\title{
In-vitro bioequivalence, physicochemical and economic benefits study for marketed innovator and generic ciprofloxacin hydrochloride tablets in Saudi Arabia
}

\author{
Ahmed F. Hanafy \\ Assistant professor of Pharmaceutics and Pharmaceutical Technology, College of Pharmacy, Taibah University, Madina, Saudi Arabia.
}

\begin{tabular}{|c|c|}
\hline ARTICLE INFO & ABSTRACT \\
\hline $\begin{array}{l}\text { Article history: } \\
\text { Received on: } 21 / 03 / 2016 \\
\text { Revised on: } 11 / 06 / 2016 \\
\text { Accepted on: } 17 / 07 / 2016 \\
\text { Available online: } 26 / 09 / 2016\end{array}$ & $\begin{array}{l}\text { The objective of the present study was to compare and evaluate the economic benefits and in-vitro } \\
\text { bioequivalence of different marketed generic ciprofloxacin tablets against the innovator tablet formulation that } \\
\text { are present in the local market of Saudi Arabia. The comparative bioequivalence and physicochemical study of } \\
\text { five ciprofloxacin marketed tablets were performed through the assessment of the uniformity of weight, } \\
\text { hardness, disintegration, dissolution, and content assay of the products. In order to compare the dissolution }\end{array}$ \\
\hline $\begin{array}{l}\text { Key words: } \\
\text { Ciprofloxacin tablets, } \\
\text { comparative dissolution } \\
\text { profile, difference factor, } \\
\text { similarity factor, } \\
\text { physicochemical parameters. }\end{array}$ & $\begin{array}{l}\text { generic products, these deviations caused significant differences in disintegration time and dissolution profile in } \\
\text { only one of the generic products. All tested generic products passed USP monograph dissolution testing except } \\
\text { one product which failed to pass both similarity factor }(f 2) \text { and difference factor }(f 1) \text { tests required by FDA } \\
\text { bioequivalence testing. The majority of generic products in Saudi Arabia which had lower prices showed } \\
\text { comparable quality to innovator. }\end{array}$ \\
\hline
\end{tabular}

\section{INTRODUCTION}

Ciprofloxacin is a synthetic fluoroquinolone derivative with broad spectrum antibacterial activity. It is widely used in the treatment of lower respiratory tract infections, urinary tract infections, skin and soft tissue infections, bacterial diarrhea, bone and joint infections, and in surgical prophylaxis (Mandell et al., 2002). Ciprofloxacin is considered to be the drug of choice by most physicians in most of the cases. This caused continuous increase in demand and accordingly the need to increase supply of generic products of ciprofloxacin in the market. It is a general psychology that the quality of generic products is considered poor when compared to leading innovator products available in the market (Adegbolagun et al., 2007).

\footnotetext{
* Corresponding Author

Ahmed F. Hanafy, Assistant professor of Pharmaceutics and

Pharmaceutical Technology, College of Pharmacy, Taibah University, Madina, Saudi Arabia.Email:drafathy@gmail.com
}

World Health Organization (WHO) has continuously supported the use of generic drug products, aiming to improve the overall health care system (Dunne et al., 2013). The generic substitution can be considered when a generic product of an innovator drug contains identical amounts of the same active ingredient in the same dose, same dosage form and route of administration together with meeting standards for strength, purity, quality, and identity (Meredith et al., 2003).

The generic products are usually much cheaper than its innovator as generic manufacturers do not have similar investment costs for the development of a new drug. In order to substitute the innovator with generics while keeping therapeutic efficacy, dissolution testing can be used as a tool to distinguish between acceptable and unacceptable drug products (Shahnaz Usman et al., 2014). It can be used as a sign for bioequivalence; dissolution testing is a practical and economic approach that can be used when there are limitations for performing in vivo studies (Meredith et al., 2003). 
In the present study, in vitro bioequivalence of some generic ciprofloxacin $\mathrm{HCl}$ tablets (500) $\mathrm{mg}$ were investigated in comparison to an innovator product of ciprofloxacin $\mathrm{HCl}$ tablets $(500 \mathrm{mg})$ to justify the quality of generic substitution of ciprofloxacin generic products in the Saudi Arabia market. Some of general quality assessments testes for tablets were also determined to access similarity between innovator brand and the different generic substitutions.

\section{MATERIAL AND METHODS}

\section{Material}

Ciprofloxacin HCl, USP (United States Pharmacopoeia, 2012) was kindly donated from Jamjoom pharmaceuticals. Brand (innovator) and four generic ciprofloxacin $500 \mathrm{mg}$ tablet products were randomly collected and purchased from Community pharmacies in Madina, Saudi Arabia. Reagents utilized including hydrochloric acid, potassium dihydrogen phosphate, and sodium hydroxide were of analytical grade.

\section{Price deviation and product label information}

The percentage price differential of four generic brands in reference to innovator was calculated using proposed formula by Akinleye et al. (Akinleye et al., 2012)

$$
\% \text { price differential }=\frac{(\text { Price of innovator }- \text { Price of generic }}{\text { Price of innovator }} \times 100
$$

Label information including manufacture date, expiry date and price per 10 tablets were compared for generic and innovator products to study if price correlates with quality of product. The innovator and generic products were purchased from market provided that they have a minimum 6 month before expiry date.

\section{Physicochemical Parameters and moisture content measurement}

Tablet dimensions (length, width and/or diameter) and hardness were measured using tablet hardness tester (Tablet hardness tester TBH 125, Erweka, Germany) using six tablets form each of the studied products, the average and standard deviation were recorded for the studied innovator and the four generic products. Disintegration and friability testing for studied tablets were performed using disintegration tester (Disintegration tester model, Pharma Test, Germany) and tablet friability tester (Friability tester model, Pharma Test, Germany) respectively according to USP.

Moisture content was measured after grinding tablets in a mortar and accurately weighing $1 \mathrm{gm}$ in aluminum plates (W1). Samples were tested at $75^{\circ} \mathrm{C}$ for 30 minutes using Halogen moisture analyzer (Halogen moisture analyzer, HB43-S, Mettler Toledo, Germany).

Assay for drug potency of studied tablet products was performed by analyzing three tablets from each brand by dissolving one tablet in $0.01 \mathrm{~N} \mathrm{HCl}$. This was followed by filtering, diluting sample and measuring spectrophotometrically at $276 \mathrm{~nm}$ (UV-Vis spectrophotometer, SP-3000 plus, Optima Tokyo, Japan).

\section{Differential Scanning Calorimetry (DSC) for studying drug- excipients compatibility}

Incompatibility between drug and excipients can alter the stability and bioavailability thereby, affecting their safety and/or efficacy. DSC (Differential scanning colorimetry, Maia DSC 200 F3, Netzsch, Germany) was used as a tool for screening compatibility of excipients used in the different generic products with ciprofloxacin as well as studying similarity of excipients used to excipients used by innovator.

Two tablets were crushed from each of the studied products. $10 \mathrm{mg}$ of each sample was sealed in aluminum crucible and tested using a heating rate of $10^{\circ} \mathrm{C} / \mathrm{min}$ at a temperature of 0 $360^{\circ} \mathrm{C}$.

\section{Fourier transformation Infrared spectroscopy (FTIR) for studying similarity of excipients between innovator and studied generic products}

Spectroscopic analysis was carried out with FTIR-total reflectance method (Fourier transformation infrared spectroscopy, IR Affinity-1, Shimatzu, Japan) to investigate similarity of excipients between the brand and generic products. Spectroscopic analysis was performed for pure ciprofloxacin $\mathrm{HCl}$, crushed innovator tablet, and crushed generic product tablets.

\section{Comparative Dissolution profile}

Dissolution test of ciprofloxacin tablets was performed according to USP ciprofloxacin tablet monograph dissolution method for innovator and the four generic products. Dissolution test was carried out using dissolution tester equipped with USP paddle (apparatus-2) operated at $50 \mathrm{rpm}$ (Dissolution tester, DT 600 , Erweka, Germany). The dissolution medium is $900 \mathrm{ml} 0.01 \mathrm{~N}$ $\mathrm{HCl}$ kept at $37 \pm 0.5^{\circ} \mathrm{C}$. Six tablets from each product were tested. $5 \mathrm{ml}$ sample were withdrawn at 5, 10, 15, 20, $30 \mathrm{~min}$, and the volumes withdrawn, replaced with fresh dissolution medium. Samples were then filtered, diluted and measured spectrophotometrically at $276 \mathrm{~nm}$ (UV-Vis spectrophotometer, SP3000 plus, Optima Tokyo, Japan).

\section{Dissolution profile data analysis}

Dissolution comparison methods recommended by FDA (United States Food and Drug Administration) guidance for immediate release solid dosage forms were used in this study (Guidance for Industry: Immediate Release Solid oral Dosage Forms, 1995). A simple model independent approach including difference factor (f1) and a similarity factor (f2) were used to compare dissolution profiles between innovator and the four generic products.

The difference factor (f1) calculates the percent (\%) difference between the two curves at each time point and is a measurement of the relative error between the two curves 
(Guidance for Industry: Dissolution Testing of Immediate Release Solid Oral Dosage Forms, 1997):

$$
f l=\left\{\left[\Sigma_{\mathrm{t}=1}{ }^{\mathrm{n}}|\mathrm{Rt}-\mathrm{Tt}|\right] /\left[\Sigma_{\mathrm{t}=1}{ }^{\mathrm{n}} \mathrm{Rt}\right]\right\} \bullet 100
$$

where (n) is the number of time interval points, (Rt) is the dissolution value of the innovator at time $(\mathrm{t})$, and $(\mathrm{Tt})$ is the dissolution value of the generic product under test at time $(\mathrm{t})$.

The similarity factor (f2) is a logarithmic reciprocal square root transformation of the sum of squared error. It is a measurement of the similarity in the percent (\%) dissolution between the two curves (Guidance for Industry: Dissolution Testing of Immediate Release Solid Oral Dosage Forms, 1997).

$$
f 2=50 \cdot \log \left\{\left[1+(1 / \mathrm{n}) \Sigma_{\mathrm{t}=1}^{\mathrm{n}}(\mathrm{Rt}-\mathrm{Tt})^{2}\right]^{-0.5} \cdot 100\right\}
$$

Curves can be considered similar when (f1) values are close to 0 , and (f2) values are close to 100. (f1) values from (0-15) and (f2)values from (50-100) ensure similarity or bioequivalence of the two curves and the performance of the product under test and innovator product (Guidance for Industry: Immediate Release Solid oral Dosage Forms, 1995).

\section{RESULTS AND DISCUSSION}

\section{Price deviation and product label information}

Product label information and price percentage differences for the innovator and four generic ciprofloxacin marketed products are presented in Table (1). Reviewing the manufacture date and expiry date for the marketed products showed large differences in labeled expiry date period. The longest expiry date period was 4 years and it belonged to the innovator and one of the generic products (Cipro-4). The other three generic products showed variability in expiry date period varying from 2 years to 3 years. These differences in expiry date period might give an indication that most of the generic products do not have the same stability achieved by the innovator product.

The percentage price differences showed that the price of ciprofloxacin innovator is higher than all generic products in Saudi market with differences varying from $38.6 \%$ to $67.3 \%$. (Cipro-4) had the lowest percentage price difference from innovator of
$38.6 \%$, the closest price to innovator. While (Cipro-5) had the highest price difference form innovator of $76.3 \%$, the lowest price between the studied generic products.

\section{Physicochemical Parameters}

Dimensions, shape, color and packaging description for studied ciprofloxacin tablet products are presented in table (2). The studied products have oblong shape and have close dimensions to the innovator except for one product (Cipro-3) which was round. This might represent a difficulty for patients to swallow (Cipro-3) as the tablet is quite big. Color for all ciprofloxacin products under study were white except for one product (Cipro-4) which was coated in blue. Tablet color might have a positive or negative psychological effect on patients (Augsburger et al., 2008), so change of this color without a reference might have a negative effect on patients. All tested ciprofloxacin tablets were scored except (Cipro-2) product. This kind of scoring is usually used to give half dose if needed, and so (Cipro-2) product would deprive patients from taking half dose $250 \mathrm{mg}$ if required. The packaging materials of the products under study are transparent packaging material except for (Cipro-3) which has an opaque packing material. Weight variation, hardness, disintegration, friability, moisture content, and content percent of the tested products are presented in Tablet (3). Weights of generic products were close to innovator product approximately $770 \mathrm{mg}$ except for (Cipro-4) which had weight of approximately $830 \mathrm{mg}$. This means that (Cipro-4) contains approximately $63 \mathrm{mg}$ excess inactive ingredients which might have negative effect in retarding disintegration time (Al Ameri et al., 2012). (Cipro-2) product tablets also showed deviation from innovator weight. It had approximately $77 \mathrm{mg}$ lower weight than innovator.

Hardness, friability, moisture content and assay results for all studied products were similar to the innovator. Disintegration time results for the generic products were all similar to innovator giving approximately 1 minute disintegration time except for (Cipro-4) tablet which had approximate disintegration time of 6 minutes. This 5 fold increase in disintegration might have a negative impact on dissolution results for tablets and it might be attributed to using different manufacturing technique like using wet granulation instead of direct compression, using different excipients, and using larger amount of excipients as seen in table (3).

Table 1: Label information and percentage price differences for five different ciprofloxacin tablet (500 mg) products marketed in Saudi Arabia.

\begin{tabular}{ccccc}
\hline Product code & Mfg. date & Exp. Date & Price/10 units & \% price differences with innovator \\
\hline Cipro -1(innovator) & $1 / 2014$ & $1 / 2018$ & 101 SR & - \\
Cipro -2 & $5 / 2013$ & $5 / 2015$ & 50 SR & 50.5 \\
Cipro -3 & $7 / 2014$ & $7 / 2017$ & 37 SR & 63.3 \\
Cipro -4 & $5 / 2012$ & $12 / 2016$ & 62 SR & 38.6 \\
Cipro -5 & $1 / 2013$ & $1 / 20116$ & 33 SR & 67.3 \\
\hline
\end{tabular}

\begin{tabular}{|c|c|c|c|c|c|c|}
\hline Product code & Length (mm) & Width (mm) & Shape & Color & Scoring & Packaging \\
\hline Cipro -1 (Innovator) & 18.18 & 8.12 & Oblong & White & scored & Transparent \\
\hline Cipro -2 & 19.36 & 6.96 & Oblong & White & Not Scored & Transparent \\
\hline Cipro -3 & 13.23 & 13.23 & Round & White & scored & Opaque \\
\hline Cipro -4 & 18.16 & 8.88 & Oblong & Blue & scored & Transparent \\
\hline Cipro -5 & 18.06 & 8.05 & Oblong & White & scored & Transparent \\
\hline
\end{tabular}

Table 2: Ciprofloxacin Products dimensions, description and packaging characteristics. 
Table 3: Physicochemical parameters of five different brands of ciprofloxacin tablets 500mg.

\begin{tabular}{|c|c|c|c|c|c|c|}
\hline Product code & $\begin{array}{c}\text { weight variation } \\
(\mathrm{mg})\end{array}$ & $\begin{array}{l}\text { Hardness } \\
(\mathbf{N})\end{array}$ & $\begin{array}{c}\text { Friability } \\
(\%)\end{array}$ & $\begin{array}{c}\text { Disintegration Time } \\
\text { (min.) }\end{array}$ & $\begin{array}{c}\text { moisture content } \\
(\%)\end{array}$ & $\begin{array}{c}\text { Assay } \\
(\%)\end{array}$ \\
\hline Cipro -1(Innovator) & $766.9+3.3$ & 23.3 & 0.02 & 1.15 & 2.35 & 95.6 \\
\hline Cipro -2 & $689.5 \pm 3.5$ & 26.6 & 0.05 & 1.10 & 2.18 & 97.5 \\
\hline Cipro -3 & $767.8 \pm 3.1$ & 24.8 & 0.05 & 1.38 & 2.06 & 95.9 \\
\hline Cipro -4 & $829.7+10.1$ & 24.1 & 0.03 & 6.10 & 1.75 & 95.9 \\
\hline Cipro -5 & $781.1 \pm 9.4$ & 23.9 & 0.03 & 1.33 & 1.85 & 96.1 \\
\hline
\end{tabular}

\section{Studying compatibility of excipients using Differential Scanning Calorimetry}

DSC thermograms for pure ciprofloxacin $\mathrm{HCl}$ monohydrate, innovator (Cipro-1) and studied generic products are presented in figure (1), figure (2) and figure (3). Pure ciprofloxacin $\mathrm{HCl}$ showed two endothermic peaks, the first is a broad endothermic peak at $150{ }^{\circ} \mathrm{C}$ which represent the dehydration peak and the second endothermic peak at $325^{\circ} \mathrm{C}$ indicating ciprofloxacin $\mathrm{HCl}$ melting peak.

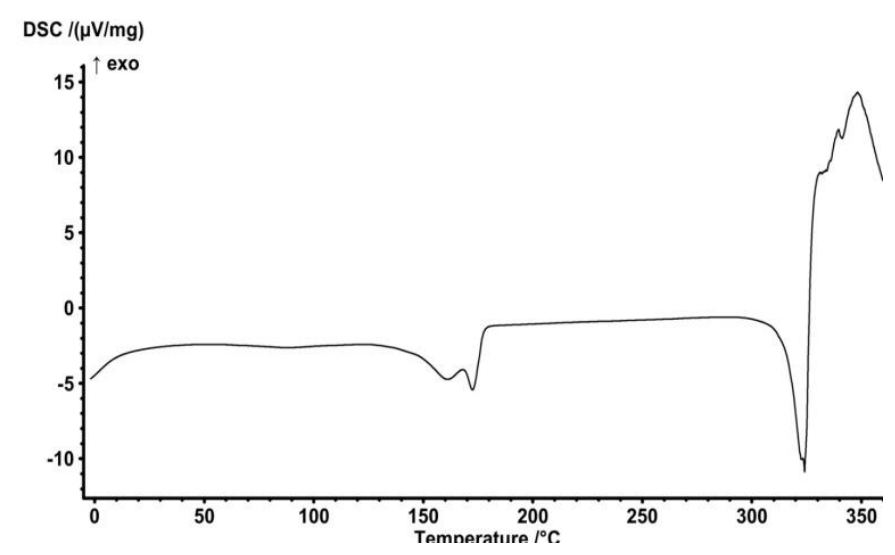

Fig. 1: DSC thermogram for ciprofloxacin $\mathrm{HCl}$ monohydrate

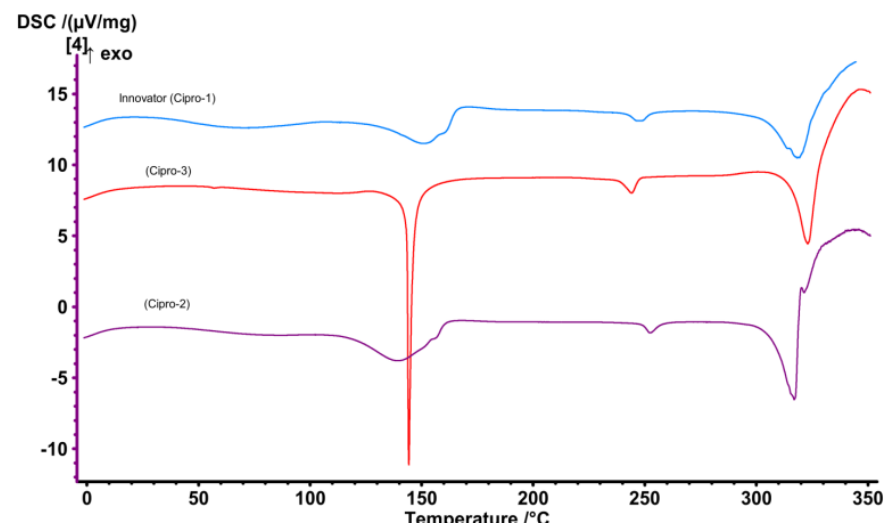

Fig. 2: DSC thermograms in sequence from upper for Cipro-1 (Innovator), Cipro-3, and Cipro-2.

These results were in accordance with Dillen et al. research work (Dillen et al., 2004). Innovator product (Cipro-1) thermograms showed three endothermic peaks, two of them are similar to pure ciprofloxacin $\mathrm{HCl}$. However, a third peak at $250^{\circ} \mathrm{C}$ appeared which might be due to one of the excipients. Generic products (Cipro-2), and (Cipro-5) thermograms showed three endothermic peaks which are very close to those of innovator. On the other hand, product (Cipro-4) thermogram showed very broad endothermic peak of dehydration which might signify different tablet preparation technique like granulation with water or hydroalcoholic solutions together with ciprofloxacin melting peak and excipient melting peak. Moreover, product (Cipro-3) showed three endothermic peaks but the second endothermic peak at $150^{\circ} \mathrm{C}$ was very sharp and long.

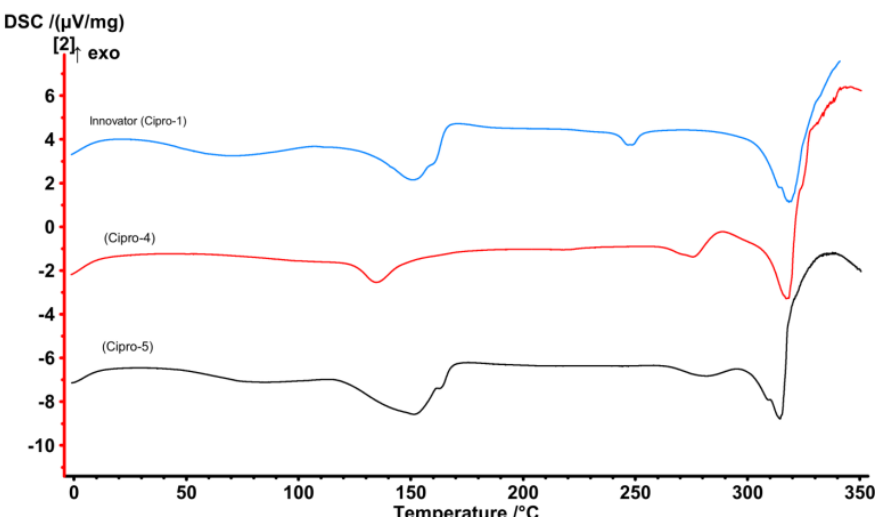

Fig. 3: DSC thermograms in sequence from upper for Cipro-1 (Innovator), Cipro-4, and Cipro-5.

This peak might be due to using another excipient in this product and not a dehydration peak as the dehydration peak is much smaller in area and broader. DSC thermogram results showed that presence of melting endothermic peak for the ciprofloxacin $\mathrm{HCl}$ in studied products' thermograms with no significant shift can indicate absence of incompatibility between ciprofloxacin $\mathrm{HCl}$ and used excipients in all products.

It should be noted that the presence of melting endothermic peaks in DSC thermogram of generic products (Cipro-3) and (Cipro-4) which are different from innovator (Cipro-1) might indicate the presence of different excipients or different tablet preparation method which might affect drug dissolution profile (Fusier et al., 2003) or cause problems for patients like allergy or any kind of intolerance (Mumoli et al., 2011).

\section{Studying similiarity of excipients using Fourier transform infrared spectroscopy}

FTIR spectra for innovator (Cipro-1) and generic products are presented in figure (4) and figure(5). FTIR spectra for the innovator (Cipro-1) seem to coincide with those of the generic 
products (Cipro-2), (Cipro-4) and (Cipro-5) which means that the innovator components might be similar to those generic products to a great extent. Generic product (Cipro-3) FTIR spectra did not coincide with that of innovator, this might signify some differences in its components.

These results were in accordance with the DSC thermogram results which showed appearance of a different sharp long peak at $150^{\circ} \mathrm{C}$.

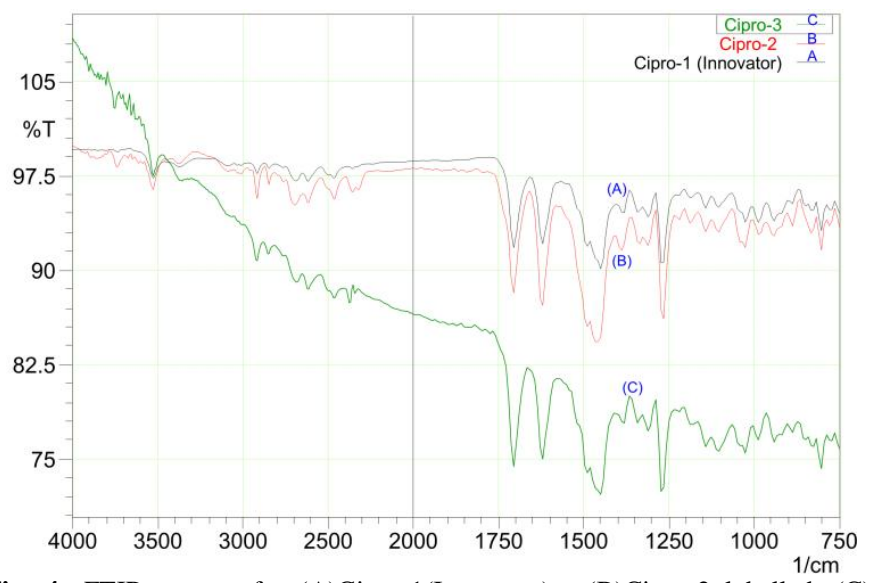

Fig. 4: FTIR spectra for (A)Cipro-1(Innovator), (B)Cipro-2 labelled, (C) Cipro-3.

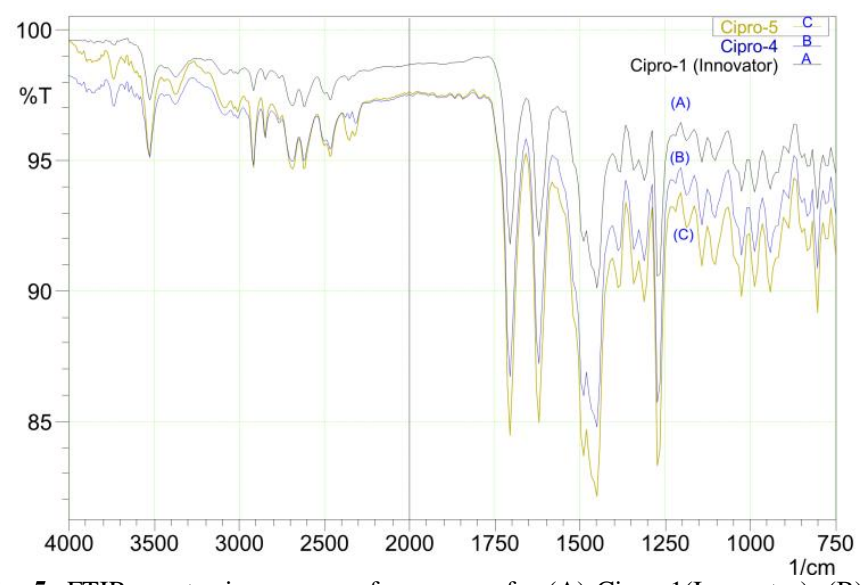

Fig. 5: FTIR spectra in sequence from upper for (A) Cipro-1(Innovator), (B) Cipro-4, (C) Cipro-5.

\section{Comparative Dissolution profile}

Dissolution profile of the innovator and four generic products are presented in figure (6). Generic product (Cipro-5) seems to be very close to innovator (cipro-1) in results in all time intervals.

The most obvious difference in dissolution is seen at the 5 minute time interval, where (cipro-2) generic product showed approximately $10 \%$ higher release than innovator, drug release $75 \%$ on average. (Cipro-3) generic product showed $10 \%$ lower drug release than innovator, drug release $65 \%$ on average. (Cipro4) release results were all much lower compared to the innovator.

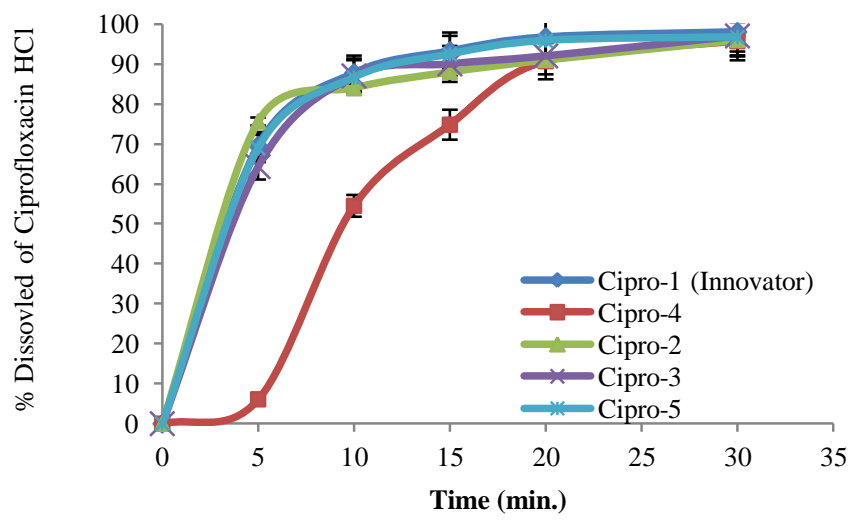

Fig.6: Dissolution rate profile in $900 \mathrm{ml} 0.01 \mathrm{~N} \mathrm{HCl}$ for innovator and four generic ciprofloxacin $500 \mathrm{mg}$ tablet products in Saudi Arabia market

\section{Dissolution profile data analysis}

Ciprofloxacin $\mathrm{HCl}$ is classified according to $\mathrm{BCS}$ (biopharmaceutical classification) as class 3 group of drugs (Kyriacos et al., 2009). The minimum requirements for accepting ciprofloxacin immediate release tablet dosage forms are specified by USP ciprofloxacin monograph. It states that the amount of not less than $80 \%$ (Q) of the labeled amount of ciprofloxacin dissolved in 30min (United states pharmacopoeia, 2012). All studied products passed this dissolution test limit.

According to the FDA guidance for industry (Guidance for Industry: Dissolution Testing of Immediate Release Solid Oral Dosage Forms, 1997), in the dissolution testing of immediate release solid oral dosage forms, the BCS suggests that for class I and in some cases class III drugs $85 \%$ dissolution in $0.1 \mathrm{~N}$ in $\mathrm{HCL}$ in $15 \mathrm{~min}$ insures that the bioavailability comply with requirement of monograph (Guidance for Industry: Immediate Release Solid oral Dosage Forms, 1995). The innovator (Cipro-1) together with 3 generic products (Cipro-2), (Cipro-3) and (Cipro-5) are passed the stated limit but only one generic product (Cipro-4) did not pass the limit after 15 minutes of dissolution as the average drug dissolution was only $75 \%$ which is $10 \%$ lower than the $85 \%$ required for complying to required bioavailability.

In order to compare the dissolution profiles of the studied innovator and generic products, a model independent approach of difference factor (f1) and similarity factor (f2) were employed (Guidance for Industry: Dissolution Testing of Immediate Release Solid Oral Dosage Forms, 1997). Similarity factor f2 has been used by FDA and the European medicines evaluation agency (EMEA) to compare the similarity of two or more dissolution profiles. For two dissolution profiles to be considered bioequivalent or similar, difference factor (f1) should be between 0 and 15 , while similarity factor (f2) should be between 50 and 100 (Guidance for Industry: Dissolution Testing of Immediate Release Solid Oral Dosage Forms, 1997). The difference factor (f1) and similarity factor (f2) values for the different generic products under study with respect to innovator (Cipro-1) are presented in table (4). The calculated values of similarity factor (f2) were more than 50 and difference factor (f1) values were less than 15 for studied products except for (Cipro-4). Thus, Cipro-2, Cipro-3 and 
Cipro-5 can be considered to be equivalent to the innovator brand. (Cipro-4) generic product had a (f2) value of 23 and (f1) value of 27. Because of the value of (f1) factor is higher than 15 and the (f2) factor is lower than 50, (Cipro-4) is considered to be dissimilar and not bioequivalent to innovator. These results were supported by the disintegration test which showed that product (Cipro-4) had much longer disintegration time relative to innovator product (Cipro-1).

Table 4: Calculated difference factor (f1) and similarity factor (f2) for all generic ciprofloxacin tablets brand in respect to Innovator (Cipro-1).

\begin{tabular}{ccccc}
\hline Studied Factor & Cipro-2 & Cipro-3 & Cipro-4 & cipro-5 \\
\hline Similarity Factor (f2) & 61 & 63 & 23 & 66 \\
Difference factor (f1) & 5 & 5 & 27 & 4 \\
\hline
\end{tabular}

\section{CONCLUSION}

Similarity of generic ciprofloxacin hydrochloride tablet products in Saudi Arabia to innovator was investigated. The physicochemical properties for all these products were studied. Some physical differences were noted in tested generic products like color, tablet scoring, tablet weight and shape. These differences from innovator might have negative impact on psychological therapeutic effect or patient compliance. Deviation in excipients or method of manufacture from innovator was noted in two generic products, these deviations caused significant differences in disintegration time and in dissolution profile in only one of the generic products. All of the tested generic products passed USP monograph dissolution testing and only one product failed to pass both similarity factor (f2) and difference factor (f1) tests required by FDA. This dissimilar generic product showed higher price when compared to other generics. While the majority of studied generic products in Saudi Arabia which had lower prices showed comparable quality to innovator to a great extent. In-vivo/ In-vitro correlation is recommended in future studies to extensively evaluate effect of changes in method of manufacturing and excipients on generic products.

\section{ACKNOWLEDGMENT}

The author would like to thank college of pharmacy, Taibah University for their support in using the research facility instruments and chemicals. The author would also like to extend his gratitude for Jamjoom Pharmaceuticals for kindly donating the active ingredient for this research work.

\section{Financial support and sponsorship: Nil.}

Conflicts of interest: There are no conflicts of interest.

\section{REFERENCES}

Adegbolagun, O. A., Osumah S. E. Comparative evaluation of the biopharmaceutical and chemical equivalence of some commercially available brands of ciprofloxacin hydrochloride tablets. Tropical journal of pharmaceutical research, 2007; 6(3): 737-745.
Akinleye, M.O, Odulaja, J.O. Comparative Evaluation of Physicochemical Properties of Some commercially available Brands of Metformin $\mathrm{Hcl}$ tablets in Lagos, Nigeria. Journal of Applied Pharmaceutical Science, 2012; 2(2): 41-44.

Al Ameri, M.N., Kumar, A., Perrett, D., Arthur Tucker, A., Johnston, A. (2012). The differences between the branded and generic medicines using solid dosage forms: In-vitro dissolution testing. Results in Pharma Sciences, 2012;2: 1-8.

Augsburger, L. L. Pharmaceutical Dosage Forms - Tablets. Baltimor, Maryland, USA, Taylor and Francis, 2008.

Dillen, K., Vandervoort, J. Factorial design, physicochemical characterization and activity of ciprofloxacin-PLGA nanoparticles. Int $\mathrm{J}$ Pharm, 2004; 275(1-2): 171-187.

Dunne, S., Dunne, C., Walter Cullen, W. A review of the differences and similarities between generic drugs and their originator counterparts, including economic benefits associated with usage of generic medicines, using Ireland as a case study. BMC Pharmacology and Toxicology, 2013; 14(1): 1-19.

Fusier I. T. C., Husson M.C. Medicines containing pharmaceutical excipients with known effects: a French review. Pharm World Sci PWS, 2003; 25(4): 152-155.

Guidance for Industry: Immediate Release Solid oral Dosage Forms, Scale-Up and Post-Approval Changes: Chemistry, Manufacturing and Controls, In Vitro Dissolution Testing, and In Vivo Bioequivalence Documentation. Rockville, MD, USA, US Food and Drug Administration, 1995.

Guidance for Industry: Dissolution Testing of Immediate Release Solid Oral Dosage Forms. Rockville,MD, USA, US Food and Drug Administration, 1997.

Kyriacos S. B. C., Saf, W., Mroueh, M., Bou Maroun, A., ElKhoury, G., Shehayeb, R. In Vitro Testing of Ciprofloxacin Formulations and Preliminary Study on BCS Biowaiver. Journal of food and drug analysis, 2009; 17(2): 78-84.

Mandell, L., Tillotson, G. Safety of fluoroquinolones: An update. The Canadian Journal of Infectious Diseases, 2002; 13(1): 54-61.

Meredith, P. Bioequivalence and other unresolved issues in generic drug substitution. Clin Ther, 2003;25(11): 2875-2890.

Mumoli, N. C. M., Luschi, R., Carmignani, G., Camaiti, A. Allergic reaction to Croscarmellose sodium used as excipient of a generic drug. QJM, 2011; 104(8): 709-710.

Usman, S. , Suleiman, R., Awad, K., Abudeek, I. Evaluation of dissolution testing for ciprofloxacin $(500 \mathrm{mg})$ tablets: post market surveillance of different brands available in Ras Al Khaimah (UAE). International journal of biopharmaceutics, 2014; 5(1): 65-72.

USP (United States Pharmacopeia, 2012.

\section{How to cite this article:}

Ahmed F. Hanafy. In-vitro bioequivalence, physicochemical and economic benefits study for marketed innovator and generic ciprofloxacin hydrochloride tablets in Saudi Arabia. J App Pharm Sci, 2016; 6 (09): 063-068. 